\title{
On Cooperative Wireless Relaying: A Joint Routing and Scheduling Flow-based Framework
}

\author{
Samat Shabdanov, Patrick Mitran, Catherine Rosenberg \\ Department of Electrical and Computer Engineering \\ University of Waterloo \\ \{sshabdan,pmitran,cath\}@uwaterloo.ca
}

\begin{abstract}
We investigate the impact of cooperative relaying used to create virtual multipoint-to-point links (as opposed to conventional multihop relaying) on the throughput optimal configuration of a wireless network. We achieve this by formulating a cross-layer framework for a joint routing and scheduling problem with cooperative relaying. We consider a general case, where cooperation is allowed between any pair of nodes in a given network. We optimally solve this formulation for max-min throughput in mesh-like networks of medium size and quantify gains for key performance metrics. We establish that, contrary to popular belief, cooperative relaying provides performance gains in a mid-size network surprisingly rarely. Moreover, if gains can be obtained, these gains are typically only marginal. We quantify these gains and provide engineering insights based on numerical results for 200 random realizations of a network with 16 nodes.
\end{abstract}

\section{INTRODUCTION}

In order to meet the increasing demand for higher data rates, next generation wireless networks must incorporate additional functionalities to enhance network throughput. Multihop networks are considered a promising approach due to their ability to extend coverage. In conventional multihop networks the data flows are routed from source to destination nodes over pointto-point links, where intermediate nodes act as decode-andforward relays. However, the use of only point-to-point links with single antennas at the transmitter and receiver does not fully exploit the benefits of the wireless medium such as spatial diversity. Cooperative relaying, which was proposed in [1] and [2], exploits spatial diversity by allowing nodes to create "virtual" multipoint-to-point links, where nodes share their antennas for simultaneous transmission. This allows a receiver to benefit from the increased Signal to Interference plus Noise Ratio (SINR) while decoding a combined signal from multiple nodes. Previous studies focus mostly on theoretical analysis of cooperative relaying for a three-node network model [3], [4].

So far, very little is known about the end-to-end throughput gains that can be achieved in any given network when cooperative relay opportunities are optimized jointly with routing and scheduling. While there are some works that address a cross-layer approach for cooperative relaying, they are based on simplified models or restricted cases. The works of [5], [6] considered only a fixed selection of relay pairs for a stringbased network with no spatial reuse during scheduling. In [7], optimization frameworks were proposed for distributed networks, but they are based on a simple protocol interference model, the use of which is questioned in [8]. A joint routing and relay assignment problem was proposed in [9], however, this model is based on the use of multiple orthogonal channels for interference-free transmission.

In this work, we neither restrict the selection of cooperative relays with which to form virtual antenna arrays nor do we consider a simplified interference model. Our goal is to quantify the performance gains of cooperative relaying on the throughput and other key performance metrics in a given network, and to provide engineering insights. We achieve this goal by formulating a generic optimization framework for joint routing and scheduling with cooperative relaying between any pairs of nodes based on a physical interference model. We mainly focus on centrally managed and fixed broadband networks with a scheduling-based access scheme that can be configured offline beforehand for optimal operation. An example of such networks is a wireless backhaul network, where base stations are connected in a mesh-like topology. For example, WiMAX and LTE are designed with options for scheduling. Users (and their mobility) are not modeled in our framework. Rate adaptation and power control are not considered in our system model.

The main contributions can be summarized as follows:

- We establish that, contrary to popular belief, the use of cooperative relaying used to create virtual multipoint-topoint links provides throughput gains in surprisingly few mid-size random networks.

- We quantify the gains on 200 random mid-size network realizations for key performance metrics such as: 1) minimum connectivity power; 2) maximum throughput gain; 3) minimum power to achieve single hop throughput. In particular, we find that while these gains are not negligible, they are usually only marginal.

- We show this by extending the formulation of [10] for the joint routing and scheduling problem with cooperative relaying. Our formulation also allows us to find an optimal configuration of a given wireless network in terms of routing and scheduling parameters without restriction on the choice of cooperative relays.

- The new formulation is of significantly larger scale than that of [10], as the maximum number of links is now of $O\left(N^{4}\right)$ (instead of $O\left(N^{2}\right)$ for [10]), where $N$ is the number of nodes. We solve this problem with the use of an efficient tool based on column generation with additional techniques to reduce the size of the problem. 


\section{FUNDAMENTALS}

\section{A. Related work}

The work of [11] was the first flow-based framework to determine an optimal throughput in any given network, regardless of the type of interference model. Prior to the work of [11], studies such as [12] focused mostly on asymptotic throughput bounds under assumptions of homogeneity of node locations. However, the approach in [11] requires to enumerate all exponentially growing number of independent sets (cliques), which makes it difficult to solve for a realistic sized network. In [13], an optimal throughput problem was decomposed into a subproblem and an integer master LP for selective enumeration of independent sets based on a column generation method. An efficient technique was proposed in [10], where independent sets are constructed with the use of a dynamic programming. The formulation of the joint routing and scheduling problem was presented in [14] for an optimal network configuration under a physical interference model. It was also shown in [14] and [10] that cross-layer optimization of routing and scheduling jointly with physical layer parameters (power and rate) provides a significant improvement in throughput over the case that does not include rate and power control. The significance of the use of the physical SINR-based interference model was discussed in [8].

The concept of cooperative relaying was first established in [15], where a three-terminal communication channel was introduced for which capacity bounds were derived. Later in [16], achievable lower bounds on capacity were obtained for a general relay channel. The use of cooperative relays via virtual distributed antennas was proposed in [1] and [2] for amplify-and-forward and decode-and-forward strategies. Outage analysis was studied in the work of [3] for these two uncoded cooperative strategies and also in [17] to compute optimal outage probability jointly with routing in networks with a string-based topology. A problem of joint resource allocation with optimal relay node selection between any source and destination pairs was studied in [18] but only for networks that are restricted to two hops.

So far, very little is known about the end-to-end performance gains that can be achieved in a given network when cooperative relaying (as opposed to conventional relaying) is optimized jointly with routing and scheduling. There exist several studies that address cross-layer design with cooperative relaying in a random access network [19] or in schedulingbased networks, however, these works are based on simplified models or restricted cases. The works of [5] and [6] considered only a fixed selection of relay pairs in a network with no spatial reuse during scheduling and with a string-based topology. Similar assumptions were used in [20] to optimize the outage probability in a string network with a fixed number of hops and mean channel gains over each path. The work of [7] proposed distributed algorithms to solve a problem of joint optimization of routing and cooperative relaying with power control, but these algorithms were derived for a simple protocol interference model. A joint routing and relay node assignment problem was formulated as a mixed-integer LP in [9], however, this model is based on the use of multiple orthogonal channels

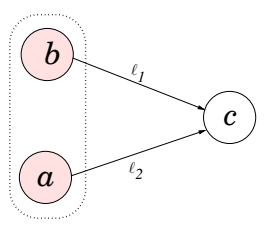

(a) without interference

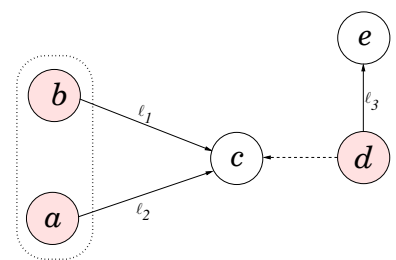

(b) with interference from $d$ to $c$
Fig. 1. Illustration of cooperative relaying

for interference-free transmission. An interesting approach of cluster-based cooperation was presented in [21], where it was shown that such a hierarchical architecture allows a network to achieve linear scaling in capacity.

\section{B. Background and Motivation}

It is well recognized that the use of multiple antennas allows a wireless network to exploit spatial diversity and to increase capacity. Multiple antennas can be employed at each node or can be distributed at different nodes in a network. We refer to the use of distributed multiple antennas via forming a "virtual" antenna array as cooperative relaying, where nodes can share their single antennas and possibly other resources for cooperative simultaneous transmission. We are interested to understand how cooperation between nodes can help to improve end-to-end network performance. We refer to Fig. 1 as a motivational example for this paper.

In Fig. 1(a), let us assume that links $\ell_{1}$ and $\ell_{2}$ are not feasible, i.e., node $c$ cannot decode signals transmitted from $a$ (resp. $b$ ) due to the Signal to Noise Ratio (SNR) $\gamma_{\ell_{1}}$ (resp. $\gamma_{\ell_{2}}$ ) over link $\ell_{1}$ (resp. $\ell_{2}$ ) being below a certain minimum threshold, say $\beta$. Thus, neither node $a$ nor node $b$ can convey a message to $c$ using only point-to-point transmission. However, it is still possible for nodes $a$ and $b$ to deliver a message to $c$ if the combined SNR is such that $\left(\gamma_{\ell_{1}}+\gamma_{\ell_{2}}\right) \geq \beta$. At node $c$, signals from $a$ and $b$ can be spatially combined with the use of standard techniques such as beamforming [2] or distributed space-time block codes [1]. Both these techniques require nodes $a$ and $b$ to transmit simultaneously with the same rate a common message. While beamforming can be used between more than two nodes, this technique requires perfect channel knowledge at the transmitters to synchronize the phases such that signals from $a$ and $b$ can add coherently at $c$. Space-time codes do not require perfect channel knowledge at the transmitters, but among all classes of codes only the Alamouti code can provide full-rate and it can be used only for cooperation between two nodes. In the sequel, we consider the case of cooperation between two nodes only based on the distributed Alamouti scheme as this does not require challenging phase synchronization.

The example in Fig. 1(a) shows that using cooperative relaying, nodes $a$ and $b$ are able to convey a message over links which are infeasible otherwise. However, it comes at the expense of increased interference to other nodes in the network since nodes $a$ and $b$ must transmit at the same time. On the other hand, node $c$ may be able to mitigate more harmful interference. In Fig. 1(b), node $d$ is the main interferer to 
node $c$ but its interference can be mitigated via cooperative relaying (i.e, $a$ and $b$ sending to $c$ together) due to the increased power of the received signal at node $c$. From this example, we can see that cooperation may improve network performance by providing better connectivity and interference mitigation, but on the other hand, it creates additional interference that may decrease spatial reuse in a network. Therefore, we aim to understand what gains the use of cooperative relaying (compared to conventional relaying) may provide in a network that is jointly optimized for routing and scheduling parameters.

\section{OPtIMIZATION FRAMEWORK}

We now propose our optimization framework based on the notion of virtual nodes that we define next.

\section{A. Assumptions and Definitions}

We model a wireless network as a set of virtual nodes $\mathcal{V}$, a set of flows $\mathcal{F}$ and a set of feasible links $\mathcal{L}$. We assume that the network is homogeneous, i.e., all nodes are of the same type and transmit with the same power $P$. We consider a network where nodes can only transmit over a single channel with a single rate $r$ corresponding to a modulation scheme with a decoding threshold $\beta(r)$. We also assume that each node is equipped with an omni-directional antenna and links are halfduplex.

We denote by $\mathcal{N}$ the set of physical nodes. A physical node represents the actual physical node in the network. Each virtual node $n$ in $\mathcal{V}$ can be either a physical node, defined by a singleton $n=\{a\}, a \in \mathcal{N}$, or a cooperative node, defined by an unordered pair of distinct physical nodes $n=\{a, b\}, a \neq b \in \mathcal{N}$. The pair $\{a, b\}$ indicates that two nodes $a$ and $b$ participate jointly in cooperative transmission to convey a common message. We allow cooperation between any two physical nodes, therefore, the set $\mathcal{V}$ must include all unordered pairs over $\mathcal{N}$, i.e., $\mathcal{V}=\{\{a, b\}: a, b \in \mathcal{N} ; a \neq$ $b\} \cup\{\{a\}: a \in \mathcal{N}\}$.

Each flow $f$ in $\mathcal{F}$ is specified by an ordered pair of physical nodes $f=\left(f_{s}, f_{d}\right)$ and $f_{s} \neq f_{d}$, where $f_{s}$ and $f_{d}$ are origin and destination of the flow $f$, respectively. Each flow $f$ in $\mathcal{F}$ can only originate at a physical node $f_{s} \in \mathcal{N}$ and can only be destined to a physical node $f_{d} \in \mathcal{N}$.

Let us define a link $\ell$ by an ordered pair of virtual nodes $\ell=\left(n_{s}, n_{d}\right)$, where $n_{s}$ and $n_{d}$ are the origin and destination nodes of the link $\ell$. We define $o(\ell)$ and $d(\ell)$ as mapping operators from the link $\ell$ to virtual nodes of origin and destination, respectively. A link $\ell$ is feasible if in the absence of interference it meets the following conditions:

$$
\begin{aligned}
& \text { [C1] } \quad d(\ell) \backslash o(\ell) \neq \emptyset, \\
& \text { [C2] } \forall j \in d(\ell) \backslash o(\ell): \frac{P \sum_{i \in o(\ell)} G_{i, j}}{N_{0}} \geq \beta(r) .
\end{aligned}
$$

The condition [C1] states that any feasible link $\ell$ must have at least one physical node in $d(\ell)$ that is not in $o(\ell)$. If all nodes in $d(\ell)$ are in $o(\ell)$, then the link $\ell$ is meaningless in the sense that all physical nodes in $d(\ell)$ already have the message intended for transmission from $o(\ell)$. The condition [C2] requires each physical node in $d(\ell)$ that is not in $o(\ell)$ to meet the minimum threshold $\beta(r)$ to successfully decode a message at the rate $r$ from $o(\ell)$. The nodes in $d(\ell)$ that

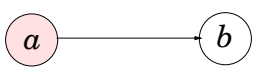

(a) $\ell=(\{a\},\{b\})$

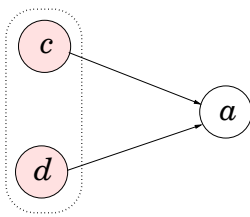

(c) $\ell=(\{c, d\},\{a\})$

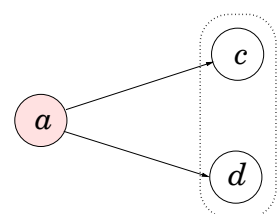

(b) $\ell=(\{a\},\{c, d\})$

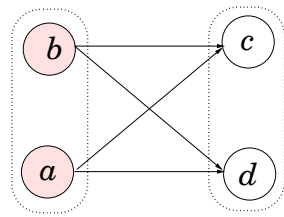

(d) $\ell=(\{a, b\},\{c, d\})$
Fig. 2. Illustration of types of links in a network

are shared with $o(\ell)$ do not need to meet [C2] as these nodes already have the message sent by $o(\ell)$. The parameters in [C2] are as follows: $N_{0}$ is the thermal noise and $G_{i, j}$ is the power gain of the channel between physical nodes $i$ and $j$, i.e,

$$
G_{i, j}=P L\left(d_{i, j}\right) \cdot g_{i, j},
$$

and is thus an aggregation of the fading gain $g_{i, j}$ and the path loss $P L\left(d_{i, j}\right)$ at distance $d_{i, j}$. In [C2], the signal power at each physical node in $d(\ell)$ is now cooperatively combined from all nodes in $o(\ell)$, e.g. using the Alamouti scheme. Let the set of feasible links $\mathcal{L}$ be a set of ordered pairs over $\mathcal{V}$, i.e. $\mathcal{L}=\left\{\left(n_{s}, n_{d}\right): n_{s}, n_{d} \in \mathcal{V} ; \ell=\left(n_{s}, n_{d}\right)\right.$ must meet [C1] and $[\mathbf{C 2}]\}$

The notion of virtual nodes allows us to incorporate all types of links in a network without restrictions on the choice of pairs of nodes participating in cooperative relaying unlike in [5], [6]. Fig. 2(a) shows a link between two physical nodes also called a basic link. Fig. 2(b) and Fig. 2(c) show links between a cooperative node $\{c, d\} \in \mathcal{V}$ and a physical node $\{a\} \in \mathcal{N}$, and Fig. 2(d) shows a link between two cooperative nodes. The origin and destination in Fig. 2(b) and Fig. 2(d) may share a physical node, e.g., $a=d$.

\section{B. Conflict-free Scheduling with ISets}

A conflict-free schedule activates at a given time a set of links that can transmit concurrently without causing harmful interference to each other. A set of links that can be spatially reused on the same channel is called an ISet. A set of links $s \subseteq \mathcal{L}$ is an ISet iff each link $\ell \in s$ meets the following conditions:

[C3] for all $\ell_{1}, \ell_{2} \in s, \ell_{1} \neq \ell_{2}: o\left(\ell_{1}\right) \cap o\left(\ell_{2}\right)=\emptyset$,

[C4] for all $\ell_{1}, \ell_{2} \in s, \ell_{1} \neq \ell_{2}: d\left(\ell_{1}\right) \cap d\left(\ell_{2}\right)=\emptyset$,

[C5] for all $\ell_{1}, \ell_{2} \in s, \ell_{1} \neq \ell_{2}: o\left(\ell_{1}\right) \cap d\left(\ell_{2}\right)=\emptyset$,

[C6] for all $\ell \in s: \forall j \in d(\ell) \backslash o(\ell)$

$$
\frac{P \sum_{i \in o(\ell)} G_{i, j}}{N_{0}+P \sum_{\substack{\ell^{\prime} \in s \\ \ell^{\prime} \neq \ell}} \sum_{i \in o\left(\ell^{\prime}\right)} G_{i, j}} \geq \beta(r) .
$$

Conditions [C3] - [C5] specify the half-duplex requirements that no two distinct links in an ISet $s$ can share a physical node of origin or destination. The condition [C6] 
requires each physical node in $d(\ell)$ that is not in $o(\ell)$ to meet the minimum SINR threshold $\beta(r)$ to allow error-free transmission with the rate $r$ over all links $\ell$ in an ISet $s$. In [C6], the co-channel interference at the receivers on link $\ell$ must be taken into account from all physical nodes of origin of all links in an ISet $s$ that are not in $o(\ell)$.

Denote by $\mathcal{I}$ a collection of all ISets and by $\alpha_{s}$ a fraction of time an ISet $s \in \mathcal{I}$ is scheduled, then the conflict-free schedule can be characterized by a time vector $\boldsymbol{\alpha}=\left[\alpha_{s}\right]_{s \in \mathcal{I}}$. Since, $\alpha_{s}$ is a fraction of time in one schedule cycle then $\sum_{s \in \mathcal{I}} \alpha_{s}=1$. Specifically, an ISet $s$ is scheduled if $\alpha_{s}>0$, otherwise $\alpha_{s}=0$.

\section{Problem formulation}

Denote by $x_{f}(\ell)$ the amount of flow $f$ transmitted over a link $\ell$ and by $R_{f}$ the rate of flow $f$. Also, denote by $\boldsymbol{x}=\left[x_{f}(\ell)\right]_{\ell, f}$ an aggregated routing vector of all flows allocated over all links and by $\boldsymbol{R}=\left[R_{f}\right]_{f \in \mathcal{F}}$ a vector of flow rates. We further assume that the traffic is static or quasi-static to enable a flow-based formulation. We define the throughput performance as a $\max -\min$ throughput $R$ since as discussed in [10], it is a reasonable objective function in centrally managed networks. The joint routing and scheduling with cooperative relaying problem is then formulated as follows [14]:

$$
\begin{aligned}
& {[\mathbf{P} 1]: \quad \quad \max _{\boldsymbol{\alpha}, \boldsymbol{x}} R} \\
& \sum_{\substack{\ell \in \mathcal{L} \\
o(\ell)=n}} x_{f}(\ell)-\sum_{\substack{\ell \in \mathcal{L} \\
d(\ell)=n}} x_{f}(\ell)=\left\{\begin{array}{cll}
R_{f}, & n=f_{s} & \\
-R_{f}, & n=f_{d} & \forall n \in \mathcal{V} \\
0, & \text { else } & \forall f \in \mathcal{F}
\end{array}\right. \\
& r_{\ell} \sum_{s \in \mathcal{I}} \alpha_{s} \mathbf{1}_{\{\ell \in s\}} \geq \sum_{f \in \mathcal{F}} x_{f}(\ell) \quad \forall \ell \in \mathcal{L} \\
& \sum_{s \in \mathcal{I}} \alpha_{s}=1 \\
& R_{f} \geq R \geq 0 \\
& \boldsymbol{\alpha}, \boldsymbol{x}, \boldsymbol{R} \geq 0 \text {. }
\end{aligned}
$$

Condition (3) specifies the flow conservation constraints for each virtual node and flow in a network. At any other virtual node the flow $f$ must be conserved along its multipath route. Constraints in (4) restrict the total amount of flow that can be scheduled over a link $\ell$ to its link capacity $r_{\ell}$. Since, we assume all nodes are enabled with a single modulation scheme, the link rate $r_{\ell}=r$ for all $\ell$ in $\mathcal{L}$. The constraint (5) states that the ISets must be scheduled over a unit period of time.

The formulation for the joint routing and scheduling problem without cooperative relaying, denoted as [P2], is identical to problem [P1], except that the set of virtual nodes $\mathcal{V}$ is now replaced by the set of physical nodes only.

The maximum number of links in a network with $N$ physical and $N(N+1) / 2$ virtual nodes is of the order of $O\left(N^{4}\right)$ and the maximum number of ISets is of the order $O\left(2^{|\mathcal{L}|}\right)$. Certainly, it is not tractable to solve [P1] and [P2] by enumerating all elements of $\mathcal{I}$, i.e., by checking for each of the $2^{|\mathcal{L}|}-1$ elements of the power set of $\mathcal{L}$ if it is an ISet or not. We solve the problems [P1] and [P2] using the column generation method to avoid the extensive enumeration of all ISets [10]. The problems [P1] and [P2] are solved iteratively and at each iteration a new subset of ISets is added into the master program. The search for ISets is performed dynamically based on their reduced costs and on other additional parameters for faster convergence to an optimal solution. If no ISets with strictly positive reduced costs can be found, then the current solution is optimal. The reduced cost for an ISet is $-\left(\zeta+\sum_{\ell \in s} v_{\ell}\right)$, where $v_{\ell}$ and $\zeta$ are the dual variables for constraints (4) and (5), respectively.

\section{Numerical Results AND ENGINEERING Insights}

In this section, we provide numerical solutions for 16-nodes mesh networks comprising of a single gateway $G W$ and $N=$ 15 other physical nodes. Typically, in a mesh network each node has a flow destined to the gateway (i.e., an uplink flow) and from the gateway to each node (i.e., a downlink flow). The results for [P1] are denoted by JRS-CR and for [P2] by JRS. We denote by $R^{*}$ the total $\max -\min$ throughput per node, i.e., $R^{*}=2 R$ since problems [P1] and [P2] are solved for $R$ assuming uplink and downlink flows at each node. The results for $R^{*}$ are obtained for $\beta_{1}=3 \mathrm{~dB}$ corresponding to a rate $r_{1}=1$ and $\beta_{2}=10 \mathrm{~dB}$ corresponding to a rate $r_{2}=2$.

Both problems [P1] and [P2] are solved for 200 random mesh networks with $N=15$ nodes and one gateway. We place nodes uniformly at random in a $2 \mathrm{~km}$ by $2 \mathrm{~km}$ square with the gateway in the center of the square. For each network realization, we modeled the constant channel gains between any pairs of physical nodes as a combination of Rayleigh flat fading with path loss. As for the path loss in (1) we use the following model [22]:

$$
P L(d)= \begin{cases}\left(\frac{\lambda}{4 \pi d}\right)^{2} & d<d_{0} \\ \left(\frac{\lambda}{4 \pi d_{0}}\right)^{2}\left(\frac{d}{d_{0}}\right)^{\mu} & d \geq d_{0}\end{cases}
$$

where $\mu=-3.3$ is the path loss exponent, $\lambda=0.3 \mathrm{~m}$ is the wavelength and $d_{0}=30 \mathrm{~m}$ is the reference distance of near field. The fading gains $g_{i, j}$ are modeled as exponentially distributed power gains with unit variance. The thermal noise is $N_{0}=-100 \mathrm{dBm}$.

The solutions for 200 networks show that cooperative relaying, contrary to popular belief, often does not improve throughput in a given random network. Fig. 3 shows the empirical CDF for the maximum relative throughput gain $G$ of JRS-CR over JRS for 200 networks where $G=$ $\max \left(\frac{R_{C R}(P)-R_{J R S}(P)}{R_{J R S}(P)}\right) \times 100 \%$. The medians of $G$ for $\beta=$ $3 \mathrm{~dB}$ is $7 \%$ and for $\beta=10 \mathrm{~dB}$ is $12 \%$. Clearly, the gains are marginal at best. Indeed, the CDF of $G$ in Fig. 3 shows that in a random network, there is a $10 \%$ probability that cooperative relaying does not provide any gain at all and "high gains" above $30 \%$ can be expected in only $10 \%$ of the cases for both $\beta=3 \mathrm{~dB}$ and $\beta=10 \mathrm{~dB}$ thresholds.

Hence, in some networks cooperative relaying does provide gains in throughput as well as it improves other key network metrics, though the improvements then are only marginal. To illustrate, among the 200 networks we selected two networks, Net-A and Net-B, shown in Fig. 4, with results that have 


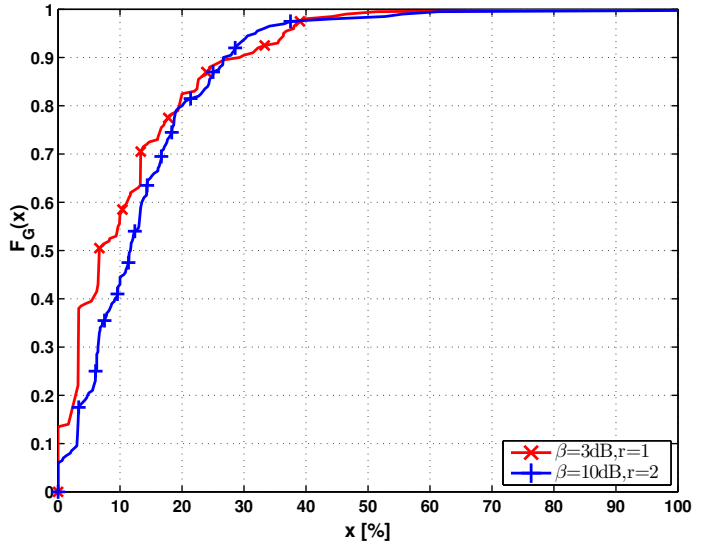

Fig. 3. CDF of the maximum relative gain $G$.

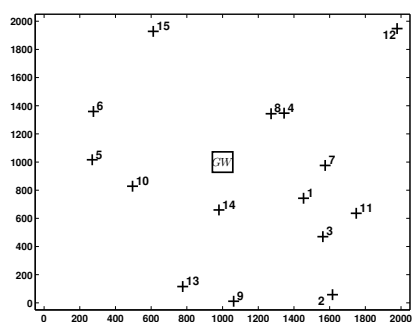

(a) Net-A

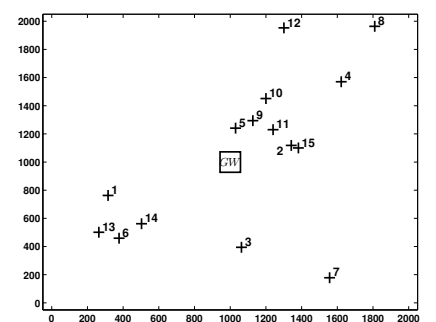

(b) Net-B
Fig. 4. Topologies of two 16-nodes networks ( $G W$ denotes the gateway).

significantly different outcomes. In the following, we denote by $P_{S H}$ the minimum transmission power at which each node is able communicate with the gateway in single hop. Fig. 5 shows $R^{*}$ as a function of the transmit power for Net$A$, a case where JRS-CR does not provide any gain at all with respect to JRS for both $r=1$ and $r=2$. In this network with the given fading realizations, the use of only the basic links is sufficient to provide the optimal $\max -\min$ throughput. On the other hand, Fig. 6 shows the case for Net- $B$ where JRS-CR provides throughput gains across low and medium transmission powers for both $\beta=3 \mathrm{~dB}$ and $\beta=10 \mathrm{~dB}$. Solution for the problem [P1] (resp. [P2]) also provides us with the optimal configuration of a network in terms of routing and scheduling parameters, and in the case of JRS-CR with the optimal selection of cooperative relaying node pairs. As an example, we show in Table I the optimal routing for the downlink flow $(G W, 13)$ using JRS and JRS$\mathrm{CR}$ at the minimum power for the network to achieve the maximum throughput of $r / N$. In the case of JRS-CR, the flow $(G W, 13)$ must be routed along two cooperative relaying nodes $\{3,5\}$ and $\{1,3\}$.

We study also two other performance metrics called $P_{\min }$ and $P_{r / N}$, where $P_{\min }$ is the minimum transmit power at which a mesh network is fully connected and $P_{r / N}$ is the minimum transmit power at which a network can yield the maximum throughput $r / N . \Delta P_{\min }$ is the difference between the minimum transmission power at which connectivity is possible when cooperative relaying is enabled and when it is

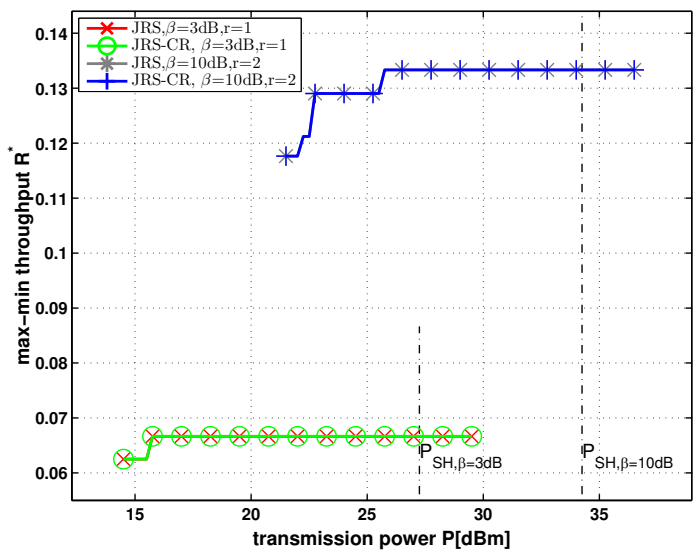

Fig. 5. $\max -\min$ throughput vs transmission power for Net-A with uplink and downlink flows.

not. Similarly, $\Delta P_{r / N}$ is the difference between the minimum transmit power at which the network can provide the maximum achievable throughput of $r / N$ when cooperative relaying is enabled and when it is not. $\Delta P_{\min }$ is a metric allowing us to quantify the gain of connectivity at low transmit power while $\Delta P_{r / N}$ allows us to quantify the gain in transmit power to obtain the same throughput as a single hop network. In Fig. 6, a gain of up to $\Delta P_{\min }=0.75 \mathrm{~dB}$ for $P_{\min }$ and $\Delta P_{r / N}=5 \mathrm{~dB}$ for $P_{r / N}$ for $\beta=10 \mathrm{~dB}$ can be achieved in the case of JRS-CR over JRS.

Fig. 7 shows the CDF of $\Delta P_{\min }$ over the 200 network realizations, where medians for cases when $\Delta P_{\text {min }}>0$ are $1.25 \mathrm{~dB}$ for both $\beta=3 \mathrm{~dB}$ and $\beta=10 \mathrm{~dB}$. In $55 \%$ of cases, cooperative relaying does not improve the minimum connectivity power at all. Also, the choice of $\beta$ does not have any impact on $\Delta P_{\text {min }}$. Gains for $P_{\text {min }}$ above $2 \mathrm{~dB}$ are infrequent and occur in less than $1 \%$ of cases. Fig. 8 shows the CDF of $\Delta P_{r / N}$ : no gains are observed in $30 \%$ of cases for $\beta=3 \mathrm{~dB}$ and likewise in $55 \%$ of the cases for $\beta=10 \mathrm{~dB}$. Gains for $P_{r / N}$ above $3 \mathrm{~dB}$ are seen in only $1 \%$ of cases for $\beta=3 \mathrm{~dB}$ and $10 \%$ of cases for $\beta=10 \mathrm{~dB}$. The medians of $\Delta P_{r / N}$ for cases when $P_{r / N}>0$ are $1.25 d B$ for $\beta=3 \mathrm{~dB}$ and $1.5 d B$ for $\beta=10 \mathrm{~dB}$. Altogether, these results show that the gains that can be obtained in mid-size networks via cooperative relaying are often not significant.

TABLE I

ROUTING IN Net- $B$ FOR DOWNLINK FLOW AT NODE 13 FOR $\beta=3 d B$.

\begin{tabular}{c|c}
\hline \multicolumn{2}{c}{ downlink flow $(G W, 13)$} \\
\hline JRS & $\{G W\} \rightarrow\{14\} \rightarrow\{13\}$ \\
\hline JRS-CR & $\{G W\} \rightarrow\{3,5\} \rightarrow\{1,3\} \rightarrow\{13\}$ \\
\hline
\end{tabular}

\section{CONCLUSions}

In this paper, we proposed a cross-layer optimization framework for joint routing and scheduling in wireless networks with cooperative relaying. This formulation is generic and we do not restrict the selection of cooperative relays nor do we 


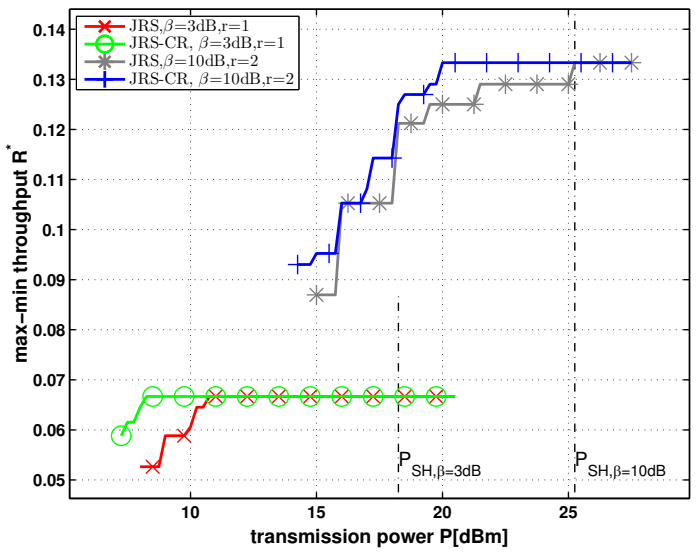

Fig. 6. $\max -\min$ throughput vs transmission power for Net-B with uplink and downlink flows.

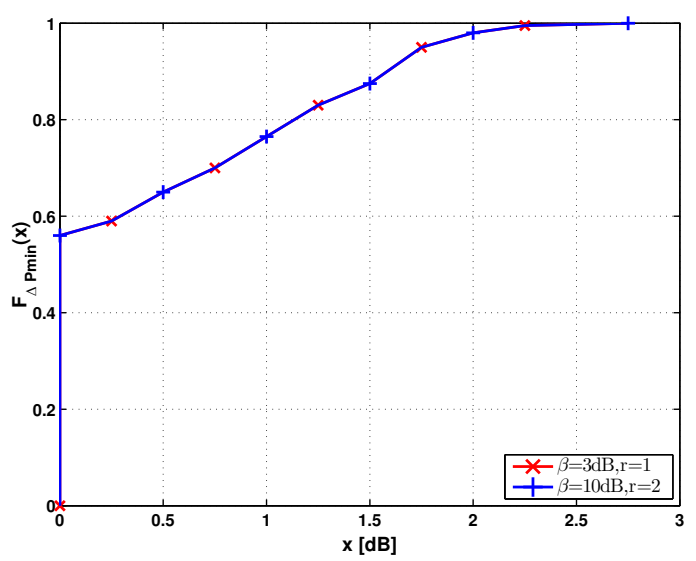

Fig. 7. $\mathrm{CDF}$ of the connectivity power gain $\Delta P_{\min }$.

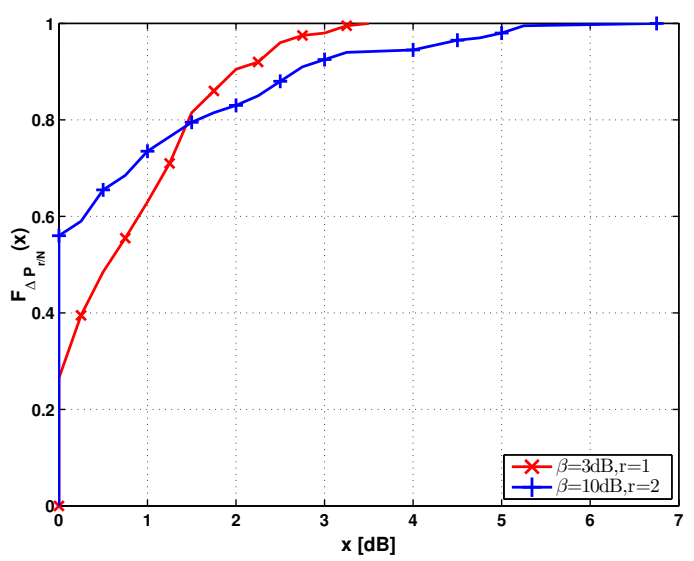

Fig. 8. CDF of power gain $\Delta P_{r / N}$.

consider a simplified interference model. We provide exact solutions for medium size networks and quantify the performance gains of cooperative relaying for a random network. Due to the complexity of the problem, results for mid-size networks with up to only 16 nodes can be computed, and therefore, we are careful to claim any conclusions on the performance of cooperative relaying in large size networks, in particular, if power control is employed. Nevertheless, our results are interesting in the sense that they allow us to contradict some popular beliefs on the benefits of cooperative relaying in wireless networks.

\section{REFERENCES}

[1] J. Laneman and G. Wornell, "Distributed space-time-coded protocols for exploiting cooperative diversity in wireless networks," IEEE Trans. Inf. Theory, vol. 49, no. 10, pp. 2415 - 2425, oct. 2003 .

[2] A. Sendonaris, E. Erkip, and B. Aazhang, "User cooperation diversity part I: System description," IEEE Trans. Commun., vol. 51, pp. 1927$1938,2003$.

[3] J. N. Laneman, D. N. C. Tse, and G. W. Wornell, "Cooperative diversity in wireless networks: efficient protocols and outage behavior," IEEE Trans. Inform. Theory, vol. 50, pp. 3062-3080, 2004.

[4] G. Kramer, M. Gastpar, and P. Gupta, "Cooperative strategies and capacity theorems for relay networks," IEEE Trans. Inf. Theory, vol. 51, no. 9 , pp. 3037 - 3063, sept. 2005.

[5] S. Cui and A. J. Goldsmith, "Cross-layer design of energy-constrained networks using cooperative MIMO techniques," Eurasip's Sig. Proc., vol. 86, pp. 1804-1814, August 2006.

[6] B. Gui, L. Dai, and L. Cimini, "Routing strategies in multihop cooperative networks," IEEE Trans. Wireless Commun., vol. 8, no. 2, pp. 843 -855 , feb. 2009.

[7] L. Le and E. Hossain, "Cross-layer optimization frameworks for multihop wireless networks using cooperative diversity," IEEE Trans. Wireless Commun., vol. 7, no. 7, pp. $2592-2602$, july 2008 .

[8] A. Iyer, C. Rosenberg, and A. Karnik, "What is the right model for wireless channel interference?" IEEE Trans. Wireless Commun., vol. 8 , no. 5, pp. $2662-2671,2009$.

[9] S. Sharma, Y. Shi, Y. Hou, H. Sherali, and S. Kompella, "Cooperative communications in multi-hop wireless networks: Joint flow routing and relay node assignment," in INFOCOM, Proceed. IEEE, 2010, pp. 1 -9.

[10] J. Luo, C. Rosenberg, and A. Girard, "Engineering wireless mesh networks: joint scheduling, routing, power control, and rate adaptation," IEEE/ACM Trans. Netw., vol. 18, pp. 1387-1400, Oct. 2010.

[11] K. Jain, J. Padhye, V. N. Padmanabhan, and L. Qiu, "Impact of interference on multi-hop wireless network performance," in Proc. MobiCom2003: Mobile Comp. and Netw. ACM, 2003, pp. 66-80.

[12] P. Gupta and P. Kumar, "The capacity of wireless networks," IEEE Trans. Inform. Theory, vol. 34, no. 5, 2000.

[13] S. Kompella, J. E. Wieselthier, and A. Ephremides, "A cross-layer approach to optimal wireless link scheduling with SINR constraints," in Military Commun. Conf.,MILCOM 2007. IEEE, oct. 2007, pp. 1 -7.

[14] A. Karnik, A. Iyer, and C. Rosenberg, "Throughput-optimal configuration of fixed wireless networks," IEEE/ACM Trans. Netw., vol. 16, no. 5 , pp. 1161-1174, 2008

[15] E. C. V. D. Meulen, "Three-terminal communication channels," Advances in Applied Prob., no. 3, pp. 120-154, 1971.

[16] T. Cover and A. Gamal, "Capacity theorems for the relay channels," IEEE Trans. Inf. Theory, vol. 25, no. 5, pp. 572-584, 1979.

[17] R. Babaee and N. Beaulieu, "Cross-layer design for multihop wireless relaying networks," IEEE Trans. Wireless Commun., vol. 9, no. 11, 2010.

[18] T. C.-Y. Ng and W. Yu, "Joint optimization of relay strategies and resource allocations in cooperative cellular networks," IEEE Trans. Sel. Commun., vol. 25, no. 2, pp. 328 -339, february 2007.

[19] P. Liu, Z. Tao, Z. Lin, E. Erkip, and S. Panwar, "Cooperative wireless communications: a cross-layer approach," IEEE Trans. Commun., vol. 13 , no. 4, pp. $84-92$, aug. 2006.

[20] A. Khandani, J. Abounadi, E. Modiano, and L. Zheng, "Cooperative routing in static wireless networks," IEEE Trans. Wireless Commun., vol. 55, no. 11, pp. $2185-2192$, nov. 2007.

[21] A. Ozgur, O. Leveque, and D. Tse, "Hierarchical cooperation achieves optimal capacity scaling in ad hoc networks," IEEE Trans. Inf. Theory, 2007.

[22] A. Molish, Wireless Communications, 2nd ed. Wiley-IEEE Press, 2005. 